\title{
Impact of the Presence of the Loop at Different Location on Fabric Width and Areal Density of a Weft Knitted Structure
}

\author{
Zubair Bin Sayed ${ }^{*}$, Nusrat Jahan ${ }^{2}$, Shabrina Kabir ${ }^{3}$ \\ ${ }^{1}$ Department of Textile Engineering, Port City International University, Chittagong, Bangladesh \\ ${ }^{2}$ Department of Textile Engineering, Ahsanullah University of Science and Technology, Dhaka, Bangladesh \\ ${ }^{3}$ Department of Textile Machinery Design and Maintenance, Bangladesh University of Textile, Dhaka, Bangladesh \\ Email: *zubinsayeed@gmail.com, nusayrk@gmail.com, shimashabrina067@gmail.com
}

How to cite this paper: Sayed, Z.B., Jahan, N. and Kabir, S. (2021) Impact of the Presence of the Loop at Different Location on Fabric Width and Areal Density of a Weft Knitted Structure. Journal of Textile Science and Technology, 7, 55-65. https://doi.org/10.4236/jtst.2021.71005

Received: December 2, 2020

Accepted: February 17, 2021

Published: February 20, 2021

Copyright $\odot 2021$ by author(s) and Scientific Research Publishing Inc. This work is licensed under the Creative Commons Attribution International License (CC BY 4.0).

http://creativecommons.org/licenses/by/4.0/

\begin{abstract}
Knitted structures are progressively built up from row after row of knitted intermeshed loops known as knitted loops. Apart from this knitted loops, tuck or miss loops may be produced by varying the timing of the intermeshing sequence of the old loop and new loops. These loops are main and prominent part of the design of the weft knitted structures. Structures having float or miss loop exhibits many noticeable characteristics. The fabrics renders better surface appearance or color pattern. Dimensional stability is significantly improved. Fabric width is reduced and areal density is also increased considerably due to the presence of float loops in the structure. Therefore, the dimensional and physical features of different knitted structures having knit-miss loops may be studied carefully to find the influence of float stitch or loop on fabric width and areal density. In this project work, such an attempt has been made to specify some specific single jersey structures, which will be ensured as an effective tool for product research and development as well as for meeting up customer's quality requirements of high class products.
\end{abstract}

\section{Keywords}

Course Per Inch, Count, GSM (Gram Per $\mathrm{M}^{2}$ ), Shrinkage, Wales Per Inch, Weft Knitting

\section{Introduction}

Knitting is a technique for developing a two-dimensional fabric made from a one-dimensional yarn or thread. It is the strategy of producing fabric by transforming ceaseless strand of yarn into arrangements of interloping loops, each 
row of such loops hanging from the one immediately preceding it [1]. The basic element of a knit fabric structure is the loop intermeshed with the loops adjacent to it on both sides and above and underneath it. Knitted fabrics are classified into two significant major groups, weft \& warp knitted fabric [1]. Weft knitted fabrics can be produced in circular or flat knitting machine. The essential knitting elements of circular knitting machines are needle, cam and sinker. The growing demand on knitted garments throughout the world appreciates the researcher to research and investigate about various knitted fabrics, production processes, developing and innovating new structures. Knitting machines comprise a needle holder that accommodates majority of needles, which are orchestrated one next to the other and can be activated with a rotating movement along their pivot as for the needle holder to shape knitting. Single cylinder circular knitting machines are commonly equipped, at the upper portion of the needle cylinder, with a precise component which is stabilized necessarily around the upper portion of the needle cylinder and given outspread cuts, inside every one of which a sinker is orchestrated and this spiral cuts are rakishly balanced around the needle chamber pivots for the needle sliding channels, so every sinker is situated between two bordering needles [2]. In the circular knitting machine with an incredible number of knitting needles, when the quantity of needle raises, the distance between the needles or sinkers have to be contracted.

Of all knitted fabrics are very much well known for its inclination and extensibility. Weft knitted fabrics are incomprehensibly utilized for day by day employments for the creation points of view, it is anything but difficult to deliver knit fabric than woven. As it is realized that yarn has been utilized for knitted fabrics needs no arrangements like warping and sizing, yarn can straightforwardly be fed on machine after receiving from spinning mills. Hand feel of knit fabrics can be adjusted by presenting miss and tuck loops on its structures where the elementary knit structures contain just knit loops. These loops straightforwardly influence the bursting strength of knit fabrics. Many researchers worked on the variation of the physical properties of weft knitted fabrics containing miss loops. Unal, P.G., et al. [3] contemplated impacts of yarn parameters, on the bursting strength of the plain knitted fabrics were analyzed with the assistance of artificial neural networks. It has been notified that the method of neural networks exhibits better concurrence with the expectation of the fabric's bursting strength. The developed neural network revealed good coincidence with the results of bursting strength.

Akaydin, M. [4] worked with the bursting strength of knit fabrics delivered from combed ring and compact yarn. It was observed that the bursting strength value of the fabrics made of compact yarn is higher than that of the fabrics made of combed yarn.

De Araújo and some prominent authors have researched mechanical conduct of weft-weaved textures for specialized applications [5] [6] [7]. It has been observed that knitted structures with longer miss loops have been recommended for improved extensibility. Thus, combining different stitch length and shaped 
miss loops a rigid or flexible structure can be achieved. Onofrei, E., et al. [8] examined the changing thermal and moisture management properties $f$ of knitted fabric structures. Single jersey, cross miss, loc knit and more couple of designs were chosen to carry out the experiment. It introduced a quantitative investigation of various comfort related properties (thermal and moisture management properties) completed on different knitted fabric structures containing Outlast ${ }^{\circledR}$ and Cool max ${ }^{\circledR}$ yarns and focusing on the choice the most satisfactory fabric for sportswear applications. Till now not so many research works have been performed on the properties of float stitches.

Some of them are referenced underneath:

Priniotakis, Charalambus \& Hadjidobrev (2004) [9] investigated the effect of float stitches on the tensile strain of both plain or rib fabrics. They also proposed a technique to forestall the breaking of the float yarn in the knitting operation due to tensile force.

Kurbak \& Kayachan (2008) [8] constructed a model of miss loop and indicated its impact on the plain knitted structure. It was additionally indicated that the model looks like the specific state of float stitch in the original fabric.

The greater part of such exploration works were focused on the presentation of miss stitch in the structure and important mechanical properties of the structures yet no work was discovered that managed the impact of settlement or accommodation of miss loop on structural property like GSM and width.

\section{Material \& Methods}

\subsection{Materials}

In this project work, the following structures are produced to evaluate the properties of weft knitted fabrics. Total number of fourteen samples was prepared based on seven different structures. This work is performed in Asha Knit Composite Mills Ltd, Dhaka, Bangladesh.

From Figure 1 we have found seven different structures varying the location of loops noted below:

(a) Structure 1-Single Jersey Plain Fabric (30 Ne CVC) (with no miss loops);

(b) Structure 2-Single Jersey Fabric with one float loop in repeat (30 Ne CVC) (with one miss loop in the repeat);

(c) Structure 3-Single Jersey Fabric with two float loops in adjacent Wales of the repeat $(30 \mathrm{Ne} C V \mathrm{C})$;

(d) Structure 4-Single Jersey Fabric with float loops in same course but not in adjacent Wales of the repeat (30 Ne CVC);

(e) Structure 5-Single Jersey Fabric with float loops in same Wales successive course of the repeat $(30 \mathrm{Ne} \mathrm{CVC}$ );

(f) Structure 6-Single Jersey Fabric with float loops in same Wales but not in successive course of the repeat ( $30 \mathrm{Ne} \mathrm{CVC}$ );

(g) Structure 6-Single Jersey Fabric with float loops in different Wales and courses of the repeat (30 Ne CVC). 


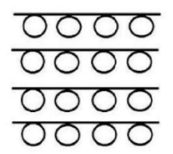

(a)

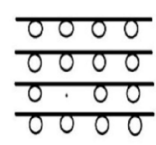

(b)

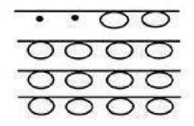

(c)

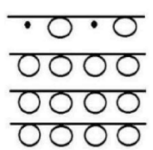

(d)

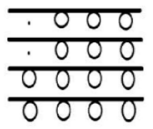

(e)

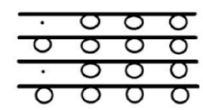

(f)

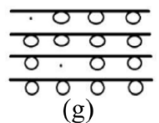

Figure 1. Different single jersey structures of different location of miss loops.

\subsection{Methods}

\subsubsection{Yarn Selection}

For the preparation of the fabrics only one type of yarn was used for this project that is $30 \mathrm{Ne} C V C(80+20)$. Only As per the requirement of the experiment the variation of properties of knitted fabric, only one type of count of yarn was used for the experimental procedure to produce six different weft knitted structures including single jersey plain fabric and putting miss loops in different position another five types of structures were produced.

\subsubsection{Machine}

Knitting machine (JIUNN LONG, TAIWAN) with 34" 24 gauges has been used. For producing six different structures different stitch length were utilized and after producing each and fabric stitch length, WPI (Wales per Inch), CPI (Course per Inch) were individually calculated.

\subsubsection{Fabric Relaxation}

Knit fabric has an exceptional property called shrinkage. At the end of the production procedure knit fabric tends to shrink. For this fabric has to undergo a standard condition for complete relaxation that means dry relaxed state relaxation. The fabrics were relaxed at $27^{\circ} \mathrm{C}$ temperature $\& 65 \%$ relative humidity.

\subsubsection{Equipment Selection}
1) Wrap reel;
2) Electric balance;
3) GSM cutter;
4) HATRA course length tester;
5) Counting glass.

\subsubsection{Determination of WPI}

After relaxation \& conditioning of knit fabric samples, the numbers of Wales in a 1" or inch length of fabric were determined by manual counting at five different places on every sample through a magnifying glass, and the average values were calculated for 30 s CVC $(80+20)$ yarn at grey state for six different structures keeping miss loops at different position.

\subsubsection{Determination of CPI}

After relaxation \& conditioning of knit fabric samples, the numbers of courses in 
a 1" or inch length of fabric were determined by manual counting at five different places on every sample through a magnifying glass, and the average values were calculated for $30 \mathrm{~s}$ CVC $(80+20)$ yarn at grey state for six different structures keeping miss loops at different position.

\subsubsection{Determination of Fabric Width}

After relaxation \& conditioning, the width of the fabric was measured by measuring tape for different samples [9].

\subsubsection{Determination of Fabric Weight (GSM)}

After relaxation \& conditioning of knit fabric samples, GSM of samples were determined with the help of GSM cutter \& weighting balance (electronic).

\section{Result \& Discussion}

\subsection{Influence of Float Loop (Due to Its Different Positions in the Structures) on Fabric Width for Different Stitch Length}

From the informative examination of Figures 2-4 it is obvious that the presence of float loop in any situation in the structure makes the fabric smaller than equal plain jersey fabric which underpins the hypothetical information. In any case, it could be seen that the width decrement \% fluctuates because of various positions of float loops in structure.

Structure having single miss (structure $b$ ) has the second most elevated width after plain jersey fabric. This is because of the presence of just one miss loop in the repeat. Here the width was diminished by around $1 \%$ than plain jersey fabric.

Structures having two float loops are smaller. It was scrutinized that the presence of two float loops reduce the width from $2 \%$ to $8 \%$ comparing with no float

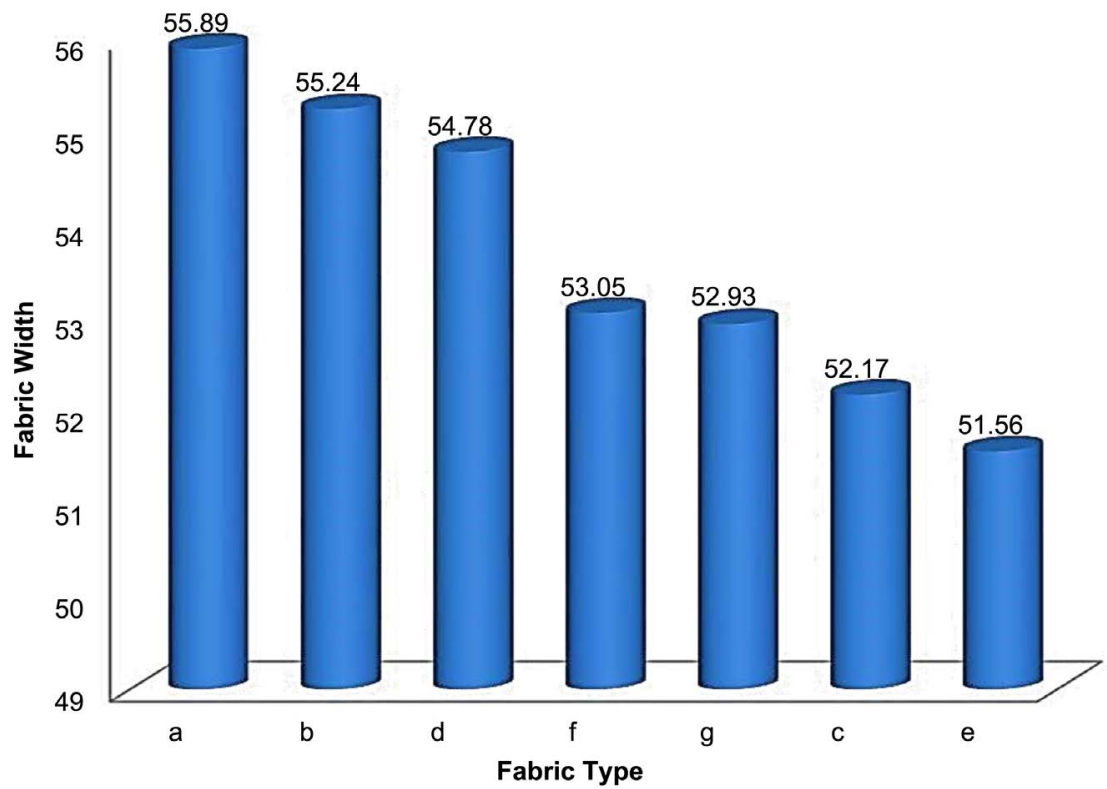

Figure 2. Comparison between fabric width and different structural fabric type of $30 \mathrm{Ne}$ CVC (Cotton + Polyester) for different average stitch length. 


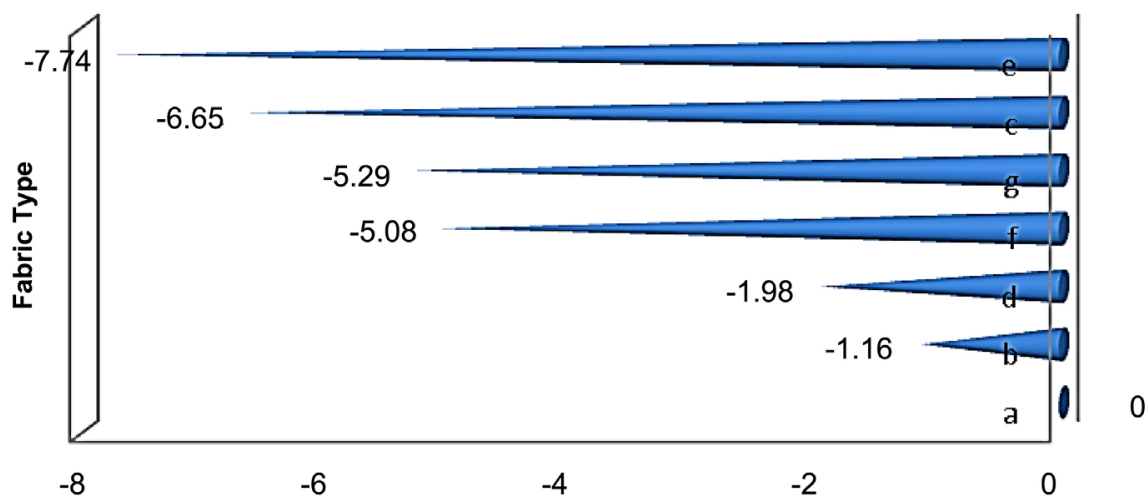

Figure 3. Comparison among different structures with respect to (a) plain jersey structure based on fabric width for different average stitch length.

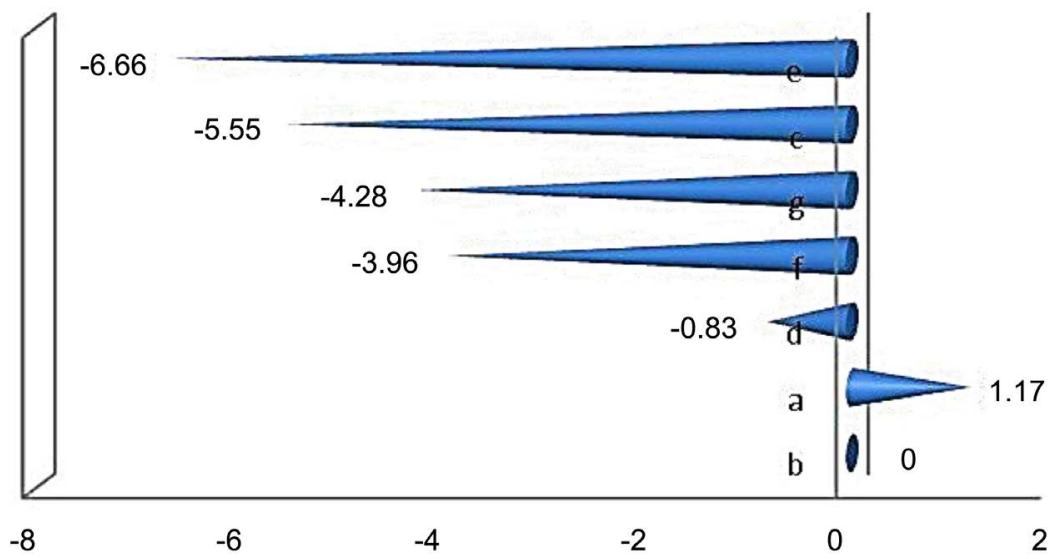

Figure 4. Comparison among different structures with respect to (b) single jersey structure with single float based on fabric width for different average stitch length.

loops in structure and this width reduction percentage ranges from $1 \%$ to $6 \%$ comparing one miss loops in the structure. From this typical comparison it is obvious that the highest width percentage is achieved for the structure $\mathrm{d}$ and the lowest one in this case is structure e.

It must be recalled that these pronouncements are appropriate for width supposition of knit miss structures with various average stitch length.

\subsection{Influence of Float Loop (Due to Its Different Positions in the Structures) on Fabric Width for Different GSM}

From Figures 5-7 it is notified that existence of float loop increases areal density. The decrement of fabric width (for example increment of W.P.I.) is the most prominent factor behind this. The contrary pattern of width graph strengthens this supposition emphatically. Single float increases GSM by $8 \%$ (generally) from float less condition. Among structures with two float loops, most elevated GSM was noticed for structure e $(46.37 \%$ higher than float less and $35.24 \%$ higher than single float structure) and least GSM was noticed for structure d (19.64\% higher than float less and $10.54 \%$ higher than single float structure). It must be 


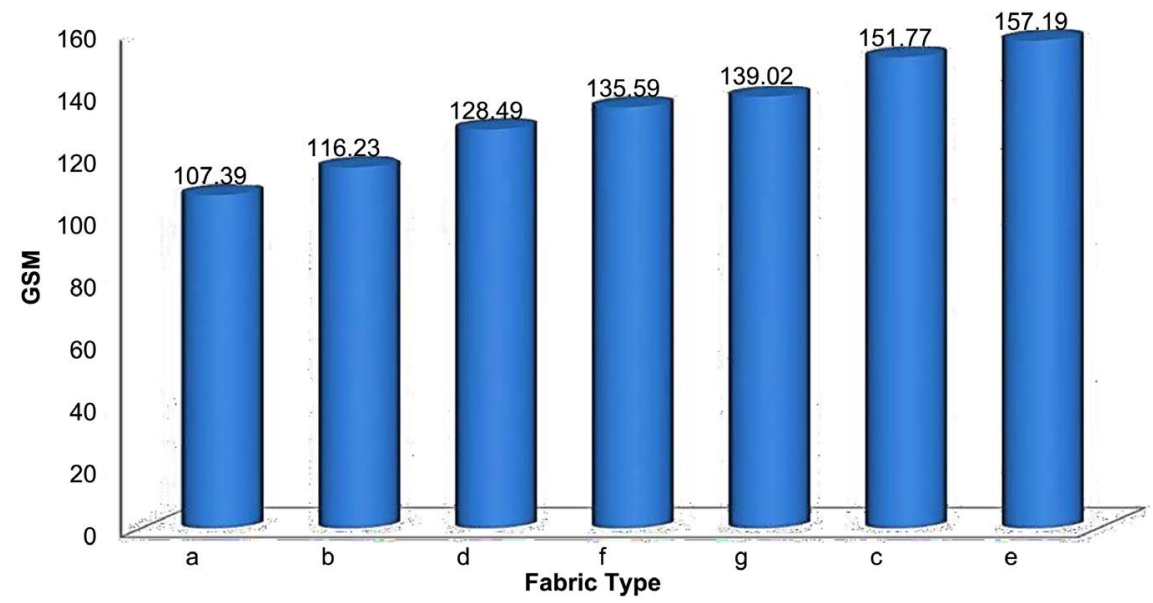

Figure 5. Comparison between fabric GSM and different structural fabric type of $30 \mathrm{Ne}$ CVC for different average stitch length.

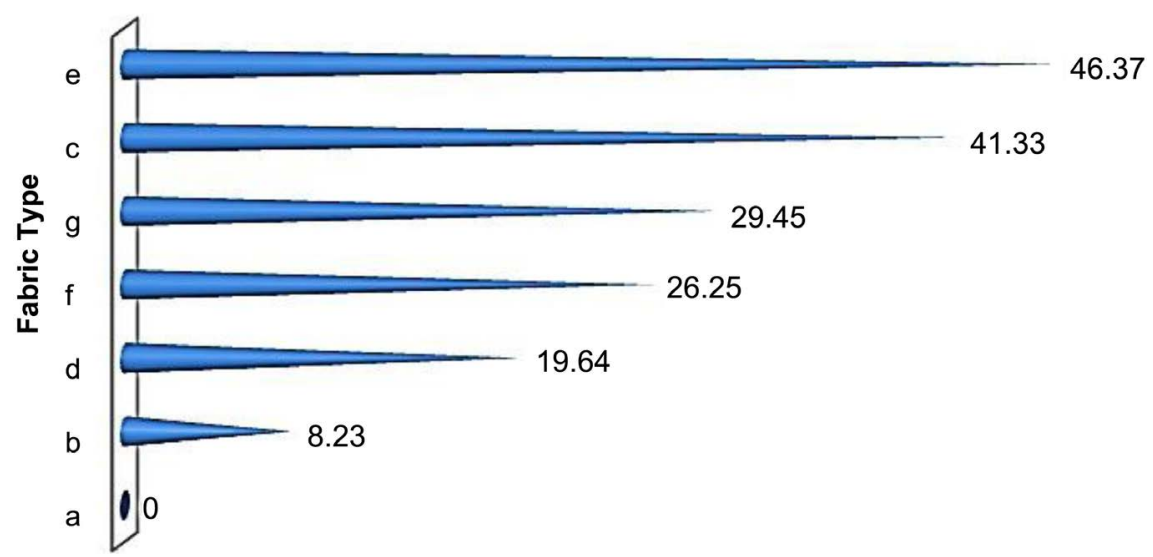

Figure 6. Comparison among different structures with respect to (a) plain jersey structure based on fabric GSM for different average stitch length.

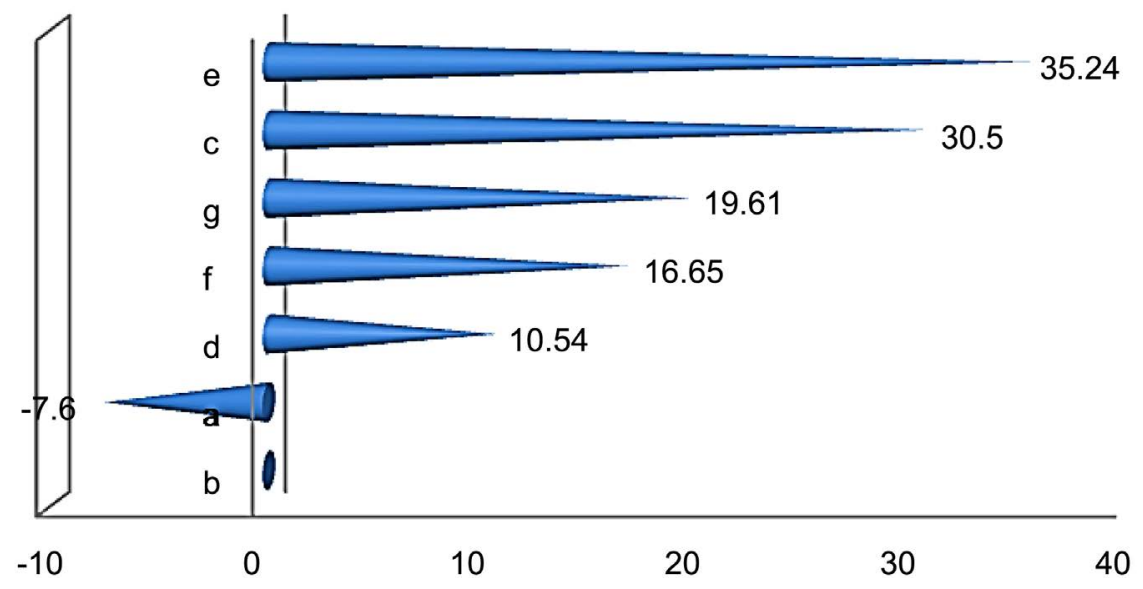

Figure 7. Comparison among different structures with respect to (b) single jersey structure with single float based on fabric GSM for different average stitch length.

recalled that these discoveries are pertinent for areal density presumption of knit miss structures with various stitch length. 


\subsection{Influence of Float Loop (Due to Its Different Positions in the Structures) on Fabric Width for Same Stitch Length}

From Figures 8-10 it is common that knit miss structures with same stitch length were produced for testing reason. Here course lengths of all knit loops were acclimated to keep up comparable S.L. (for example $2.9 \mathrm{~mm}$ ) of the structural repeat and such regulation brought about some alternation in the patterns that were noticed for normal knit miss structures with various average stitch length.

Plain jersey (no miss loop) indicated the most reduced width and the structure with single float loop demonstrated the most elevated width. Such kind of conduct

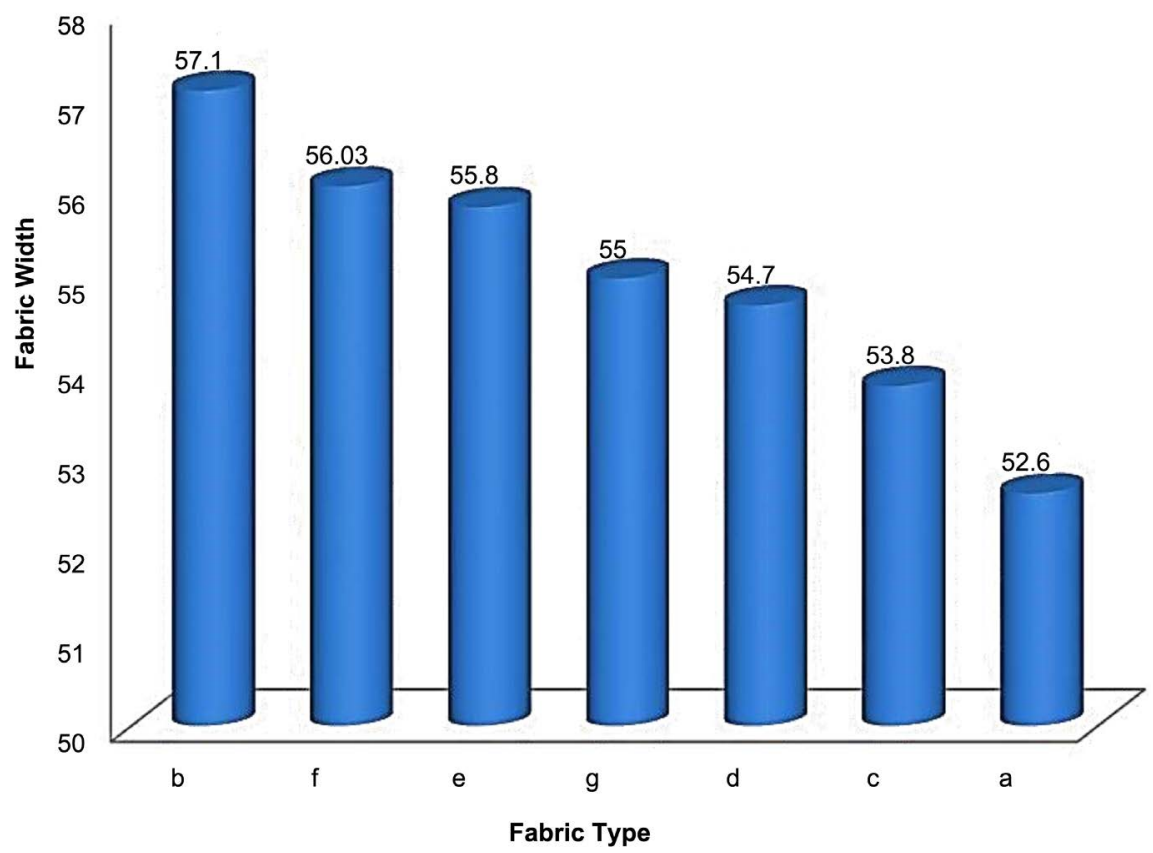

Figure 8. Comparison between fabric width and different structural fabric type of $30 \mathrm{Ne}$ CVC for same average stitch length.

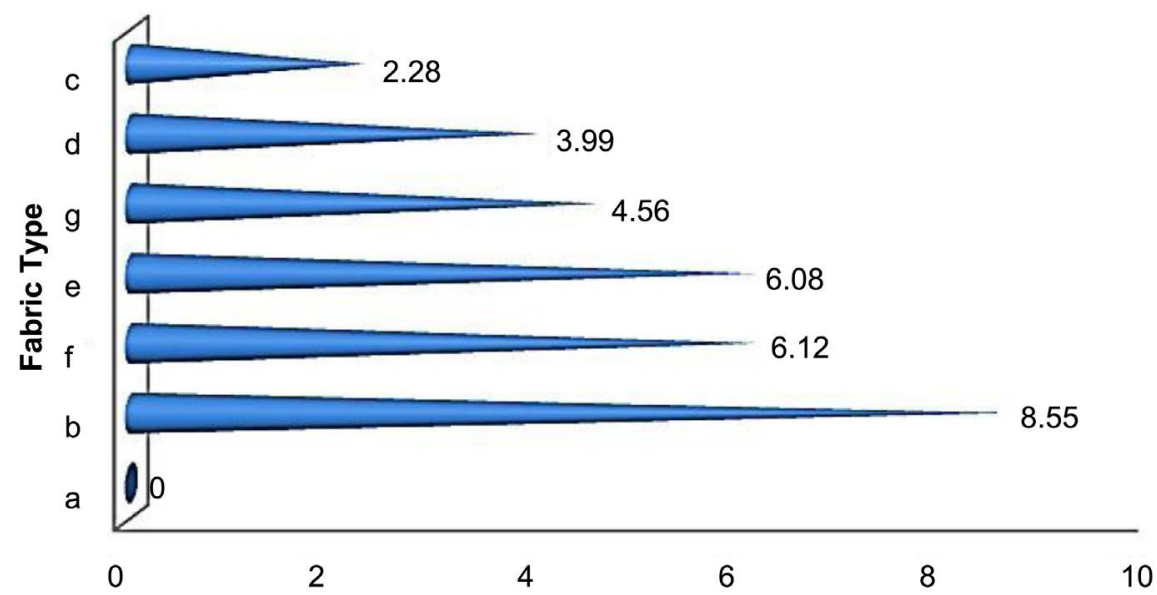

Figure 9. Comparison among different structures with respect to (a) plain jersey structure based on fabric width for same average stitch length. 
might be because of the variety of loop shape in structures, which eventually impacted W.P.I of structures.

For instance in plain jersey structure course length was similar (for example S.L $2.9 \mathrm{~mm}$ ) for all courses of the repeat however in single miss structure the knit miss course was knitted at similarly lower course length (S.L $2.4 \mathrm{~mm}$ ), the other three all knitted courses of the repeat were knitted at higher course length (for example S.L 3.07) and hence all knitted courses ruled on general basic width and made it more extensive.

\subsection{Influence of Float Loop (Due to Its Different Positions in the Structures) on Fabric GSM for Same Stitch Length}

From Figures 11-13 it might be very well examined that however normal S.L was similar for all structural repeats, GSM was not same. Variety in course separation and Wales division is the cause behind this. The contrary pattern of width graph was seen here. Structure with single float loop indicated the least GSM and the plain jersey structure (for example no miss loop) indicated the most noteworthy GSM (around 25.5\% higher than the single miss structure).

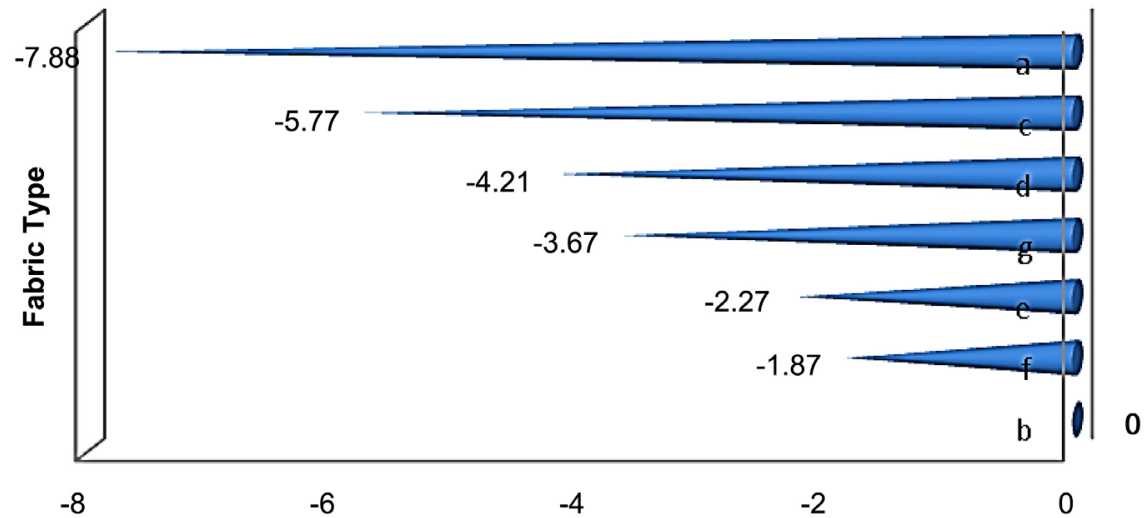

Figure 10. Comparison among different structures with respect to (b) single jersey structure with single float based on fabric width for same average stitch length.

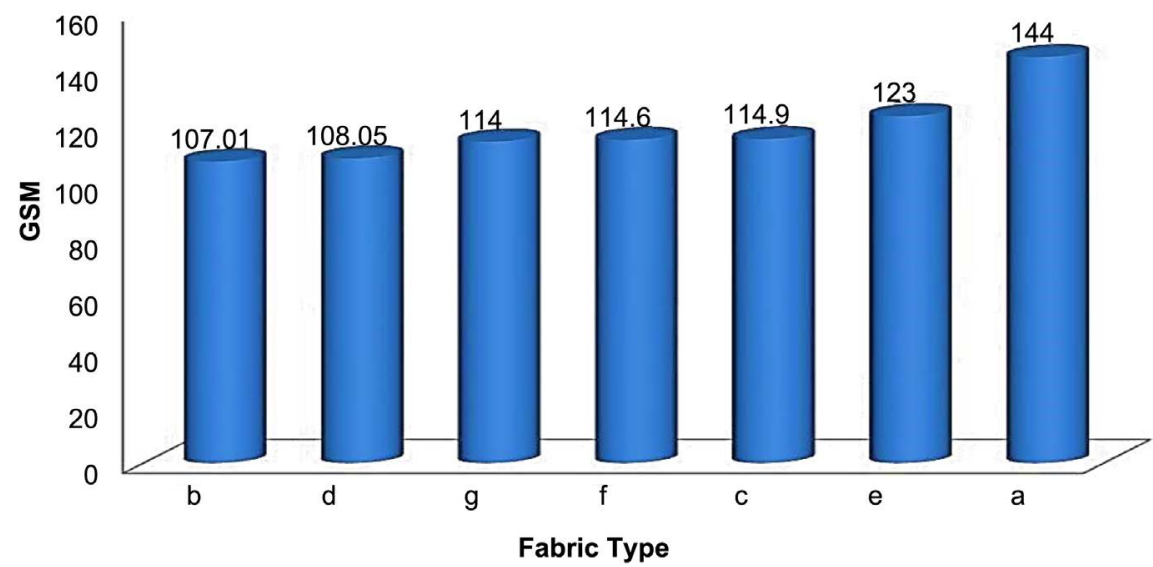

Figure 11. Comparison between fabric GSM and different structural fabric type of $30 \mathrm{Ne}$ CVC for same average stitch length. 


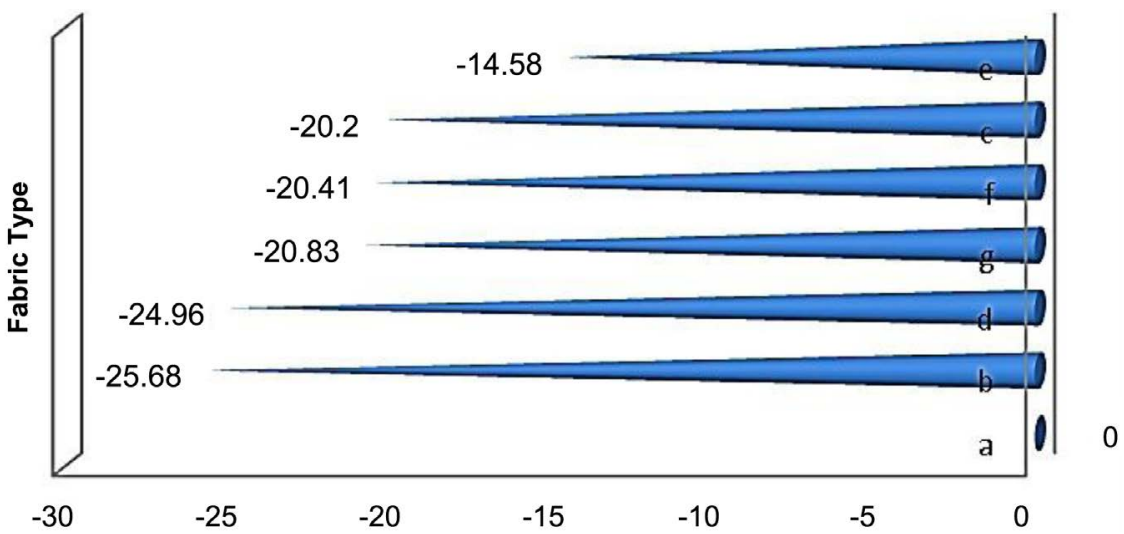

Figure 12. Comparison among different structures with respect to (a) plain jersey structure based on fabric GSM for same average stitch length.

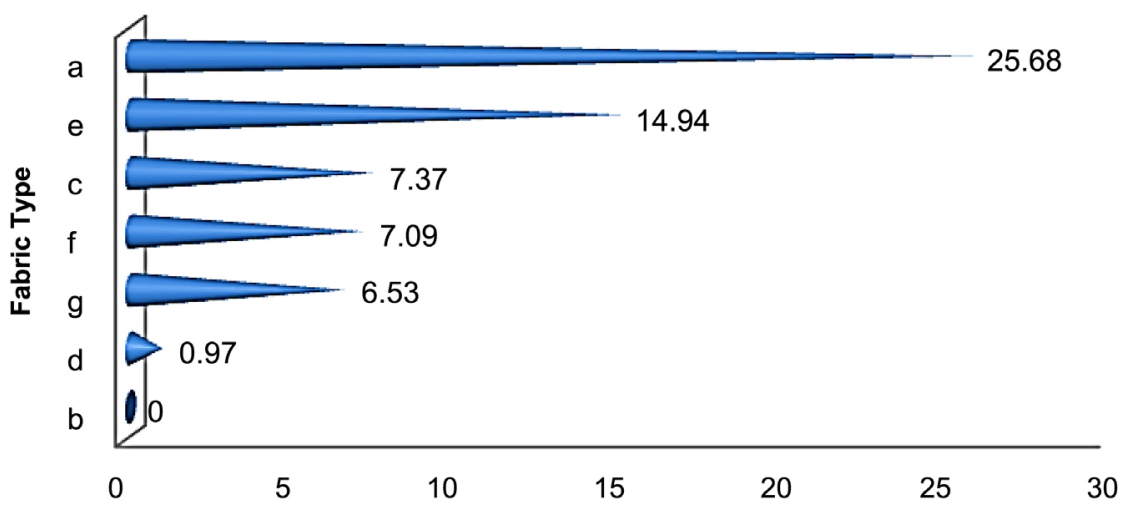

Figure 13. Comparison among different structures with respect to (b) single jersey structure with single float based on fabric GSM for same average stitch length.

\section{Conclusion}

The undertaking "Impact on texture width and areal thickness because of the presence of float loop at various situations in a weft weaved structure" is a way to deal with locates the dimensional conduct of a single jersey structure with miss or float loops. Despite the fact that the delivered structures are not so much available in mass production, their qualities might be considered for commercial production and for the launching of new products. Once more, there might be such structures in textile industry where we may discover critical areas of float or miss. Loops are more similar to the experimental structures. Thus, there is an extent of additional exploration here. We trust this task work will help us in our expert professional career.

\section{Conflicts of Interest}

The authors declare no conflicts of interest regarding the publication of this paper.

\section{References}

[1] Abedin, F., Maniruzzaman, K., Sina, A.S., et al. (2014) Effect of Gauge Variation of 
Circular Knitting Machine on Physical and Mechanical Properties of Cotton Knitted Fabrics. International Journal of Textile Science, 3, 70-75.

[2] Lonati, E., Lonati, F. and Lonati, T. (2017) Knitting Machine, Particularly with High Gauge, with Improved Needle Actuation Cams. Google Patents.

[3] Ünal, P.G., Üreyen, M.E. and Armakan, D.M. (2010) Predicting Bursting Strength of Plain Knitted Fabrics Using ANN in ICAART 2010. Citeseer.

[4] Akaydin, M. (2009) Characteristics of Fabrics Knitted with Basic Knitting Structures from Combed Ring and Compact Yarns. International Journal of Textile Science, 2, 5-11.

[5] Bekampiene, P. and Domskiene, J. (2009) Analysis of Fabric Specimen Aspect Ratio and Deformation Mechanism during Bias Tension. Materials Science, 15, 167-172.

[6] Islam, A. (2014) Effect of Wale Wise Increasing of Tuck and Miss Loops on Bursting Strength of Single Jersey Fabric at Grey and Finish State. International Journal of Research in Engineering, 3, 7-9.

[7] Mikučionienė, D., Čiukas, R. and Mickevičienè, A. (2010) The Influence of Knitting Structure on Mechanical Properties of Weft Knitted Fabrics. Materials Science, 16, 221-225.

[8] Onofrei, E., Rocha, A.M. and Catarino, A. (2011) The Influence of Knitted Fabrics' Structure on the Thermal and Moisture Management Properties. Journal of Engineered Fibers Fabrics, 6, Article ID: 155892501100600403.

https://doi.org/10.1177/155892501100600403

[9] Priniotakis, G., Charalambus, A. and Hadjidobrev, P. (2004) The Knitting Thread's Stretch at the Float Stitch with Elasticity of elongation Only. Materials Science Forum, 455-456, 775-780.

https://doi.org/10.4028/www.scientific.net/MSF.455-456.775 